\title{
Resonator properties of paranasal sinuses: preliminary results of an anatomical study*
}

\author{
Miriam Havel', Lena Ertl', Daniel Bauer², Maria Schuster' ${ }^{1}$, Klaus Stelter', \\ Johan Sundberg ${ }^{3}$ \\ Rhinology 52: 178-182, 2014 \\ DOl:10.4193/Rhino13.097 \\ ' Department of ORLHNS, Munich University Hospital, Munich, Germany \\ *Received for publication: \\ ${ }^{2}$ Chair of Medical Education, Munich University Hospital, Munich, Germany \\ July 1, 2013 \\ ${ }^{3}$ Department of Speech, Music and Hearing, School of Computer Science and Communication, KTH \\ Accepted: January 11, 2014
}

(Royal Institute of Technology), Stockholm, Sweden

\begin{abstract}
Background: The contribution of the nasal and paranasal cavities to vocal tract resonator properties is unclear as are voice effects of sinus surgery. Here we investigate resonance phenomena of paranasal sinuses with and without selective occlusion of the middle meatus and maxillary ostium in a cadaver.
\end{abstract}

Methodology: Nasal and paranasal cavities of a Thiel-embalmed cadaver were excited by sine-tone sweeps from an earphone in the epipharynx. The response was picked up by a microphone at the nostrils. Different conditions with blocked and unblocked middle meatus were tested. Additionally, infundibulotomy was performed allowing direct access to and selective occlusion of the maxillary ostium.

Results: Responses showed high reproducibility. Minor effects appeared after removal of meatal occlusion. A marked low frequency dip was detected after removal of occlusion of maxillary ostium following infundibulotomy.

Conclusion: Reproducible frequency responses of nasal tract can be derived from cadaver measurements. Marked acoustic effects of the maxillary sinus appeared only after direct exposure of the maxillary ostium following infundibulotomy.

Key words: resonance, nasal tract, paranasal sinuses, cadaveric study, functional endoscopic sinus surgery

\section{Introduction}

It is a longstanding issue that the air-filled paranasal sinuses in the facial cranium contribute substantial acoustic effects to the human voice. Such effects were first proposed in the 17 th century by Bartholinus ${ }^{(1)}$. He noted that paranasal sinuses were not present in individuals with a "faulty voice". Howell studied the Maori people in New Zealand and observed "peculiarly dead" voices in those with "an underdevelopment of their accessory sinuses" (2). However, according to other authors, there is no relation between voice quality and paranasal sinuses as these are regarded as poor resonators due to their anatomical structure (limited size of ostia, turbinates covering the ostia, sinonasal mucosa dampening vibration) ${ }^{(3,4)}$. From the surgical point of view, voice alteration following sinus surgery also seems to be a controversial issue as some authors have observed no voice effects of endonasal surgery ${ }^{(5-7)}$, whereas others have found measurable acoustical changes ${ }^{(8,9)}$.

Sinus ostia are narrow and complex anatomical structures with considerable intra- and interindividual variation, and they are difficult to visualize. Their patency as well as the patency of the nasal cavity can vary as the sinonasal tract is lined with mucosa with considerable swelling potential. A variety of exogenous factors as well as the physiological phenomenon of the endogenous nasal cycle ${ }^{(10)}$ lead to non-controllable swelling condition of the mucosa in vivo, which would substantially alter the acoustical properties of the nasal tract. 
Tepper et al. investigated the effect of sinus floor elevation on voice in four patients. No changes in the measured acoustical parameters (pitch, dynamic range, sound pressure level, jitter, shimmer and noise-to-harmonics ratio) were detected ${ }^{(7)}$. However, a comparison of voice quality in twenty-one patients, who had undergone endonasal sinus surgery, showed subjectively perceivable alterations as well as some spectral effects (decrease of bandwidths and increased energy peaks of formants) ${ }^{(8)}$. Chen and Metson also found measurable effects on the produced acoustic signal and on perceived nasality in five patients' speech following ethmoidectomy ${ }^{(9)}$. However, analyses of nasal consonants and nasalized vowels are limited in the sense that results are influenced by numerous non-controllable factors, such as anatomy, mucosa swelling, velopharyngeal opening, etc.

It has been commonly assumed that widening the ostia by means of decongestants or surgery leads not only to an alteration of the resonance but also to a stronger coupling between the nasal tract and the paranasal cavities. However, this assumption has found scarce support in investigations carried out under conditions of a stable sinonasal anatomy.

Summarizing, a problem with in vivo studies is that they may reflect various, not comparable acoustical features: the pre- and postoperative studies have been obtained following endonasal surgeries of various extents and for different sinonasal pathologies, and in many cases, results have been averaged across patients. Given the great diversity of individual nasal and paranasal morphology, it may be more promising to study effects of systematic surgical manipulations made under well-controlled, stable conditions in a single case. Therefore, in the present study we systematically examine acoustic effects of paranasal cavities in a cadaver.

\section{Materials and methods}

A Thiel-preserved human male cadaveric sinonasal tract was acoustically excited via an earphone (Jabra BT 3030, Copenhagen, Denmark) placed and hermetically sealed by plasticine in the epipharynx. Sweep tones obtained from the Tone generator ${ }^{\oplus}$-software (by Svante Granqvist, KTH, Stockholm, Sweden) were introduced into the nasal cavity. The frequency range of the sweep tone was $200 \mathrm{~Hz}$ to $4000 \mathrm{~Hz}$ with a duration 18s. The signal was picked up $2 \mathrm{~cm}$ in front of the nostrils using an electret condenser-microphone (TCM141) and recorded via Wavesurfer ${ }^{\circledast}$ audio editor (by Jonas Beskow and Kåre Sjölander, KTH, Stockholm, Sweden), sampling frequency $16000 \mathrm{~Hz}$. On endoscopic exploration using $0^{\circ}$ and $45^{\circ}$ rigid $4.0 \mathrm{~mm}$ endoscopes (Karl Storz, Tuttlingen, Germany) no pronounced septal deviation was identified and the middle meatus, sphenoidal ostia as well as preserved mucosa showed a normal anatomy as visualized and documented by means of a TelePack X-documentation device (Karl Storz, Germany). Moreover, CT scan in axial acquisition and coronal/sagittal reconstruction revealed no sinonasal pathology, anatomical anomaly or previous surgery on the investigated sinonasal tract. The examined cadaveric sinonasal tract was Thiel-embalmed. In contrast to the conventional formaldehyde-based fixation, in this cadaver preservation technique based on ethylene glycol, the preserved tissue does not harden and colour as well as the consistency of tissue resembles the natural in vivo condition (11).

Resonance properties of the nasal and sinus cavities were tested in four conditions:

A. prior to surgery

1. occlusion of both middle meatus and sphenoidal ostia

2. removal of meatal occlusion on one side

B. following surgery

3. infundibulotomy and exposure of maxillary ostium

4. occlusion of same maxillary ostium.

First, to obtain a reference condition, both middle meatus and sphenoidal ostia were occluded by endoscopically controlled placement of a viscous mass prepared of maltodextrin food thickener (Nutrilis ${ }^{\circledR}$ powder, Milupa, Germany) and water. Second, the maltodextrin occlusion of the right middle meatus was removed by targeted suction. Third, all occlusions were removed and infundibulotomy was conducted on the right side, allowing exposure of the natural maxillary ostium. Fourth, this ostium was occluded. For each condition, responses to sine-sweep excitation were measured twice.

At the time of the experiment the paranasal sinuses on the left side were almost completely filled with the fixation fluid. The occlusion of middle meatus and sphenoidal ostia on both sides as well as the manipulation solely on the right side prevented this remaining embalming fluid from interfering with the acoustical measurements.

Fundamental frequency and sound level of the recorded responses were measured via the Soundswell ${ }^{\circledR}$-software (Saven Hitech AB, Täby, Sweden) using the Corr and Extract subroutines of the Soundswell ${ }^{\circledR}$ work station. Five hundred sample points of these signals, equally spaced in time, were obtained by means of Extract and imported into Excel. This allowed plotting the recorded level data as function of frequency.

\section{Results}

Baseline experiments/ Reproducibility

The reproducibility of the sine sweep response curves was tested by repeated recordings. Figure 1 shows typical results, two response curves obtained under the same condition (both middle meatus and both sphenoidal ostia occluded). In the figure, one curve has been shifted by two decibels for the sake of demonstration. The curve shows a marked resonance at about $700 \mathrm{~Hz}$ and blunt resonances at 2700 and $3700 \mathrm{~Hz}$. As can be 
seen in the figure, discrepancy between the two recordings was negligible.

\section{Removal of the occlusion from middle meatus}

Minor spectral effects of the sine sweep response curves were observed after removal of the occlusion from the right middle meatus; an increase of about twenty $\mathrm{Hz}$ of the lowest dip and the lowest peak and some details at higher frequencies (Figure 2).

Infundibulotomy, exposure and selective occlusion of maxillary ostium

After removal of the processus uncinatus the two curves shown in Figure 3 were obtained, one with the ostium occluded, the other with the ostium open. In the latter case, a marked dip appeared near $500 \mathrm{~Hz}$.

\section{Discussion}

This would be the first attempt to experimentally examine acoustic properties of the nasal tract in isolation, without influences of nasal mucosa swellings and with well-controlled surgical modification of morphology.

We examined the nasal tract in isolation, without including the vocal tract. Thereby it was important to achieve a complete sealing of the velopharyngeal opening, as even a tiny leakage would have changed the resonance properties considerably. Under conditions of occlusion of both middle meatus and sphenoidal ostia in the cadaver, resonance peaks were observed near $700 \mathrm{~Hz}, 2700 \mathrm{~Hz}$, and $3700 \mathrm{~Hz}$. These frequencies show some similarity with those of an open-closed cylindrical resonator of $12 \mathrm{~cm}$ length: $\left[(2 \mathrm{n}-1)^{*} \mathrm{~F}=34000 \mathrm{~cm} \mathrm{~s}-1 / 4^{*} 12 \mathrm{~cm}=700 \mathrm{~Hz}\right]$. An overall nasal tract length of $12 \mathrm{~cm}$ is realistic for an adult male. As mentioned, the nasal tract is an extremely complex and unstable resonator, due to the intricate sinonasal anatomy as well as the cyclically changing mucosa shape. By using the stable anatomical condition of a cadaver, we could obtain quite reproducible response curves to the sine sweeps (Figure 1).

Assuming that the maxillary sinus can be considered as Helmholtz resonator with a neck area of $30 \mathrm{~mm}^{2}$, neck length of 1 $\mathrm{mm}$, and a volume of $20,000 \mathrm{~mm}^{3}$, its lowest resonance frequency should be close to $700 \mathrm{~Hz}$. This value is close to the frequency of the zero observed after the maxillary ostium was exposed.

To examine the acoustical contributions of the paranasal cavities in vivo, several authors have made use of a direct comparison of nasal spectra in normal and "opened ostia" condition by application of decongestants (e.g. epinephrine, xylometazoline) ${ }^{(12,13)}$. The results from these studies differ from those gained in the present investigation. However, as the inferior turbinate represents the main decongestant-responsive nasal component,

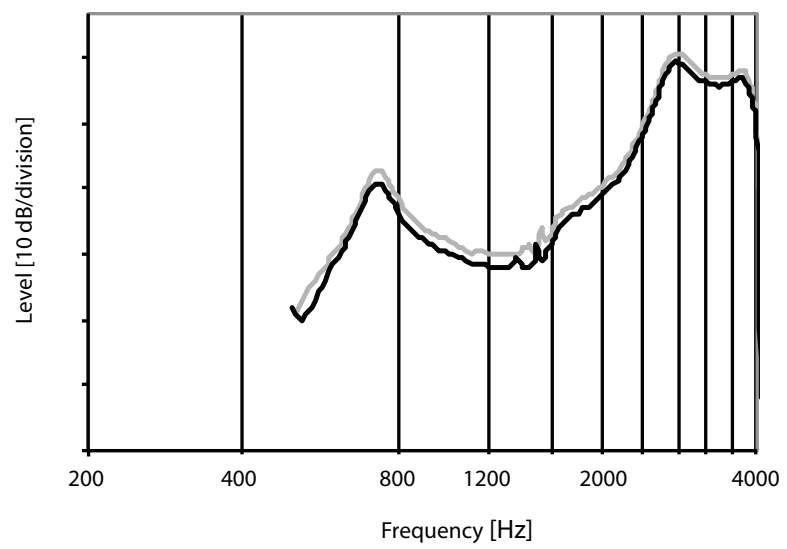

Figure 1. Response curves from two sine-sweep excitations of the nasal tract to illustrate reproducibility. In both the middle meatus and the sphenoidal ostia were occluded (reference curve). For the sake of clarity one curve was displaced by $2 \mathrm{~dB}$.

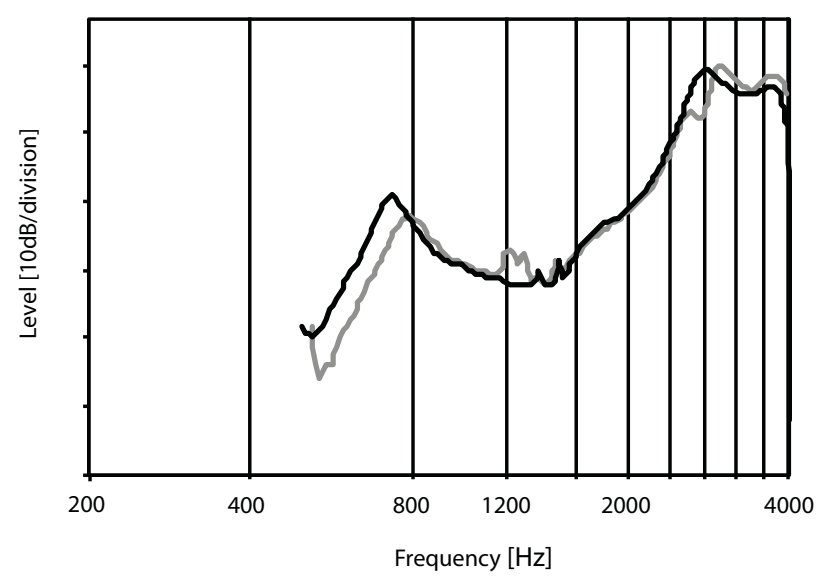

Figure 2. Response curves from sine-sweep excitation of the nasal tract. The grey curve was obtained after removal of right meatal occlusion. The black curve represents the reference condition from Figure 1.

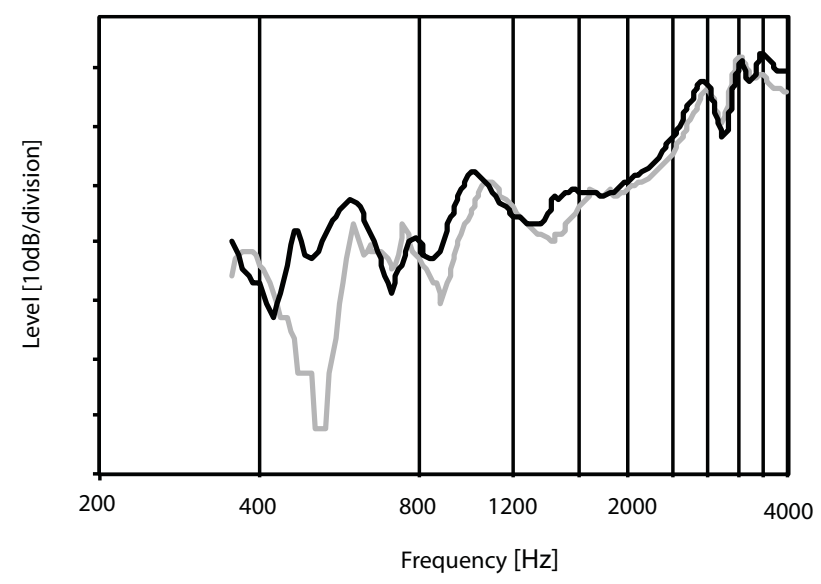

Figure 3. Response curves obtained from sine-sweeps after infundibulotomy. Grey curve shows the response prior to occlusion of maxillary ostium, black curve after occlusion. 
it is likely that the main part of the observed alterations in the spectra resulted from the volume reduction of the inferior turbinate rather than from paranasal cavities' resonance effects. Blocking the sinus ostia by filling the cavities with water or iodine-containing contrast agent (Proetz method) and additional packing with gauze bandage has been used as an alternative method to explore the acoustic relevance of paranasal cavities $(14,15)$. Applied in vivo, such an extensive manipulation is likely to cause mucosal irritation followed by swelling, which would compromise the experimental condition in an uncontrollable way.

In 1966, Koyama investigated the resonance mechanisms of the paranasal sinuses by means of a plastic model of human vocal tract coupled to maxillary and sphenoid sinus models of different volumes and ostium diameters. He observed several effects after this coupling, e.g. "a flat fall and sharp valley in the middle tone range", which he assumed to be induced by antiresonance of paranasal sinuses ${ }^{(14)}$. His experiment is comparable to ours as it used standardized conditions that affected only the ostia. As illustrated in Figure 3, we observed a clear dip in the frequency response when the maxillary ostium was exposed. However, resonance characteristics might be different due to complexity of the nasal cavity and absence of the mucosa.

Comparing normal and epinephrine-decongested nasal mucosa conditions Masuda introduced sine sweeps in the epipharyngeal space of 13 healthy subjects and picked it up at the anterior nostrils ${ }^{(16)}$. The resonance frequencies of the maxillary sinuses ranged from 1 to $2 \mathrm{kHz}$ with considerable variation between individuals. However, in compound models/computed simulation, the maxillary sinus models have been assumed to resonate below $1 \mathrm{kHz}{ }^{(12,17)}$. In these reports however, the ostium of the maxillary sinus was modelled as a straight pipe.

As pointed out by Dang and Honda the sinuses are in acoustical contact with the nasal tract. Hence they should function as acoustic absorbents, producing zeros or minima in the transfer function ${ }^{(18)}$. Due to the asymmetric shape of the cavities, such zeros may appear at more or less different frequencies. Furthermore, if the passage to a cavity is narrow and long, its acoustic impedance will be high and resistive and hence its effects on nasal sound should be small. We observed small dips in the response curves after removal of the occlusion from the middle meatus, i.e. when the maxillary ostium was still shielded from the nasal cavity by the processus uncinatus. By contrast, a clearly marked zero near $500 \mathrm{~Hz}$ appeared following removal of the occlusion from the maxillary ostium, but only when it was directly exposed after infundibulotomy. This supports the assumption that under normal conditions the maxillary and frontal sinuses will have negligible effects on the spectrum of nasals ${ }^{(18)}$. Clinical studies on voice quality changes following sinonasal surgery published so far ${ }^{(7-9)}$ have arrived at conflicting conclusions. The present work conducted in cadaveric state allowed for well-controlled conditions concerning the cyclical mucosa changes as well as the interindividual morphological diversity. However, these preliminary findings do not allow immediate transfer to clinical practise. Further, well-designed investigations are indicated to close the knowledge gap between ex vivo and in vivo findings combining clinical and acoustical expertise.

This novel combined surgical-acoustical approach to analyse sinonasal resonance properties has shown promising results. Selective occlusion of middle meatus and sinus ostia by maltodextrin mass application in a stable morphology of cadaveric state seemed a suitable method for examination of resonance effects. Minor acoustical effects were detected for maxillary sinus in the natural condition, i.e. when shielded from the nasal cavity by the processus uncinatus, but exposed by infundibulotomy, the sinus showed marked effects. The results provide evidence supporting the assumption that sinonasal surgery may have effects on the voice.

\section{Authorship contribution}

MH/JS conceptualized and designed the study. MH/LE/DB collected and assembled the data. Data analysis and interpretation were conducted by JS/MH, who also drafted the article. DB/ MS/KS critically revised the article for intellectual content. All authors approved the final version of the article.

\section{Acknowledgement}

This work received no funding.

\section{Conflicts of Interest}

The authors describe no conflict of interest.

\section{References}

1. Keir J. Why do we have paranasal sinuses? J Laryngol Otol. 2009; 123: 4-8.

2. Howell HP. Voice production from the stand point of a laryngologist. Ann Otol RhinoLaryngol. 1917; 26: 443-455.

3. Negus $V$. The function of the paranasal sinuses. AMA Arch Otolaryngol. 1957; 66:
430-442.

4. Schaeffer JP. The nose, the paranasal sinuses, nasolachrymal passageways and olfactory organ in man. Am J Med Sci. 1920; 160: 122-128.

5. Blanton PL, Biggs NL. Eighteen hundred years of controversy: the paranasal sinuses. Am J Anat. 1969; 124: 135-147.
6. Blaney SPA. Why paranasal sinuses? J Laryngol Otol. 1990; 104: 690-693.

7. Tepper G, Haas R, Schneider B, et al. Effects of sinus lifting on voice quality. A prospective study and risk assessment. Clin Oral Implants Res. 2003; 14: 767-774.

8. Hosemann W, Göde U, Dunker JE, Eysholdt $U$. Influence of endoscopic sinus surgery 
on voice quality. Eur Arch Otorhinolaryngol. 1998; 255: 499-503.

9. Chen MY, Metson R. Effects of Sinus Surgery on Speech. Arch Otolaryngol Head Neck Surg. 1997; 123: 845-852

10. Braun T, Rich M, Berghaus A, Kramer MF. Effects of oxymetazoline nasal spray on the nasal cycle assessed by long-term rhinoflowmetry. Rhinology. 2012; 50: 370-375.

11. Thiel W. The preservation of the whole corpse with natural color. Ann Anat. 1992; 174: 185-195.

12. Maeda S. The role of the sinus cavities in the production of nasal vowels. Proc. IEEE Int. Conf. Acoustics, Speech, and Signal Processing. 1982; 7: 911-914.

13. Linqvist-Gauffin J, Sundberg J. Acoustic properties of the nasal tract. Phonetica. 1976; 33: 161-168
14. Koyama T. Experimental study on the resonance of paranasal sinus. J Otolaryngol Jpn. 1966; 69: 1177-1191.

15. Vennard W. An experiment to evaluate the importance of nasal resonance in singing. Folia Phoniatr (Basel). 1964; 16: 146-153.

16. Masuda S. Role of the maxillary sinus as a resonant cavity. Nihon Jibiinkoka Gakkai Kaiho. 1992; 95: 71-80.

17. Takeuchi S, Kasuya H, Kido K. A study on the effects of nasal and paranasal cavities on the spectra of nasal sounds. J Acoust Soc Jpn. 1977; 33: 163-172.

18. Dang J, Honda K. Acoustic characteristics of the human paranasal sinuses derived from transmission characteristic measurement and morphological observation. J Acoust Soc Am. 1996; 100: 3374-3383.

\section{Dr. med. Miriam Havel}

Department of Otorhinolaryngology

Head \& Neck Surgery

Munich University Hospital

Marchioninistr. 15

81377 Munich

Germany

Tel: 0049-89-7095-3888

Fax: 0049-89-7095-6869

E-mail: miriam.havel@med.uni-

-muenchen.de 